\title{
Hard-X-Ray Lensless Imaging of Extended Objects
}

\author{
J. M. Rodenburg, ${ }^{1, *}$ A. C. Hurst, ${ }^{1}$ A. G. Cullis, ${ }^{1}$ B. R. Dobson, ${ }^{2}$ F. Pfeiffer,${ }^{3}$ O. Bunk, ${ }^{3}$ C. David, ${ }^{3}$ \\ K. Jefimovs, ${ }^{3}$ and I. Johnson ${ }^{3}$ \\ ${ }^{1}$ Department of Electronic and Electrical Engineering, University of Sheffield, Sheffield S1 3JD, United Kingdom \\ ${ }^{2}$ CCLRC Daresbury Laboratory, Daresbury, Warrington WA4 4AD, United Kingdom \\ ${ }^{3}$ Paul Scherrer Institut, CH-5232 Villigen PSI, Switzerland \\ (Received 29 September 2006; published 18 January 2007)
}

\begin{abstract}
We demonstrate a hard-x-ray microscope that does not use a lens and is not limited to a small field of view or an object of finite size. The method does not suffer any of the physical constraints, convergence problems, or defocus ambiguities that often arise in conventional phase-retrieval diffractive imaging techniques. Calculation times are about a thousand times shorter than in current iterative algorithms. We need no a priori knowledge about the object, which can be a transmission function with both modulus and phase components. The technique has revolutionary implications for $\mathrm{x}$-ray imaging of all classes of specimen.
\end{abstract}

Introduction. - Lensless x-ray diffraction microscopy is one of the most promising methods for life and material science imaging on the nanometer scale. The high scattering vectors ( $q$ values) available in the Fraunhofer diffraction plane can, in principle, yield wavelength-limited resolution (i.e., approaching the nanometer scale), without any of the limitations which apply to x-ray lenses. The key task is to solve computationally for the phase of the scattered intensity. Crystallographic methods are not applicable, given that any object of microscopic interest is, by definition, aperiodic. In the absence of periodicity, it is possible to use a size constraint, either in the object itself or in the extent of a collimated beam illuminating the object. Solution of the phase problem is then rendered tractable via certain iterative phase-retrieval methods [1-9]. Large aperiodic objects have highly structured diffraction patterns. To solve for a given field of view from a single diffraction pattern, the detector must have a pixel size (in the Fraunhofer plane) which is inversely proportional to the size of the object. In terms of practical microscopy, this is a severe limitation: Microscopists require the ability to scan a large field of view in order to place an object of interest in spatial context, preferably in real time. Current phase-retrieval imaging methods suffer from further limitations. Even if the diffraction plane sampling criterion is satisfied, a unique solution often depends upon the object being single-valued (for example, being a pure phase object or a purely absorptive object). In fact, many objects of practical interest impress both phase and modulus changes onto the exit wave field. Current methods are also computationally intensive, typically requiring tens of thousands of iterations (each involving two Fourier transforms over the entire - even if limited - field of view), meaning that any possibility of real-time imaging is remote.

Ptychographical iterative engine.-We demonstrate here an x-ray microscope which overcomes all of these limitations. The theory of the technique [10-13] has been demonstrated to work at low resolution at light optical wavelengths [14], but the scientific impact at X-ray wavelengths is revolutionary. We demonstrate a proof of principle that x-ray imaging can be accomplished without the need for any sophisticated optics. Given the imminent development of much higher brightness fourth-generation coherent sources, we believe that the method will open up the possibility of real-time, wavelength-limited hard (or soft) $\mathrm{x}$-ray imaging of objects of any size. Our experimental approach relies on collecting a number of Fraunhofer diffraction patterns (Fig. 1), each of which comes from a different, but overlapping, region of the specimen which is moved laterally across the illuminating beam. The key development is to meld all of these data into a wide field of view. The method is related to a direct (noniterative) solution of the crystallographic phase problem first proposed by Hoppe [15]. An aperture the size of the unit cell is placed in real space over a periodic object. In the Fraunhofer diffraction plane, each crystalline reflection is now convoluted with the Fourier transform of the aperture function. In one dimension, such an aperture is a "top hat"; hence, the diffracted peaks are convoluted with sinc functions. At their point of overlap, the sinc functions add according to the complex values determined by the phase of the underlying diffracted beams. The intensity of each adjacent diffracted peak is easily measured: The intensity at the midpoint (the "in-between diffraction spots," nowadays referred to as the "oversampled" diffraction spots [3]) gives a measure of their relative phase. Hoppe recognized that this measure could still lead to an ambiguity of a complex conjugate in each diffracted beam, as is usual in the classic phase problem [16]. However, by moving the aperture function to a new position displaced by less than one unit cell, a new measure can be obtained for this phase difference, and hence all phases are obtained unambiguously. This method, relying on a convolution (or "folding") in reciprocal space was later called "ptychography" 


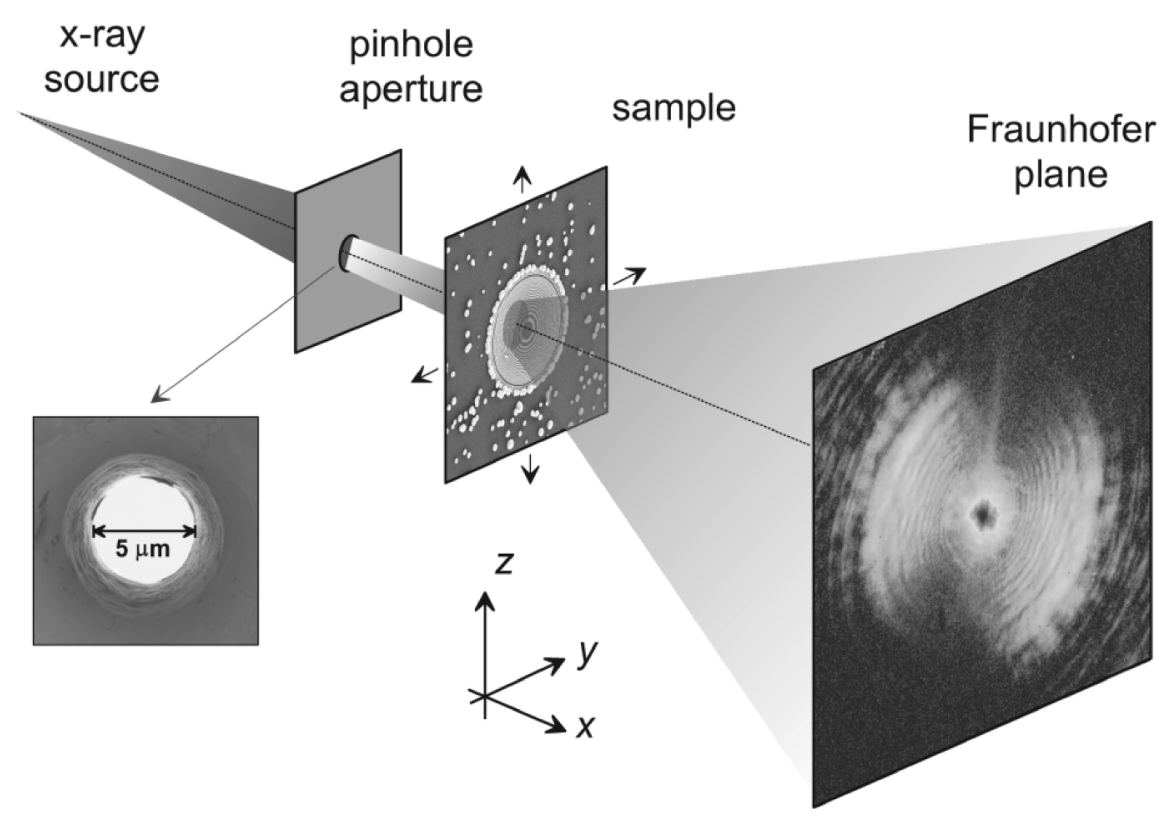

FIG. 1. Schematic of the experimental setup for a shifting specimen coherent x-ray diffraction microscopy.

(from the Greek " $\pi \tau v \xi$," meaning to fold [17]). While it may not be obvious, ptychography is helpful for extended noncrystalline objects, and, in fact, many of its benefits still apply [18]. Our iterative technique incorporates these ptychographic phase data to piece together a large field of view [10-13]. The algorithm calculates an initial exit wave $(\psi)$ for a particular aperture position on the basis of a current estimate of the object function $(O)$ :

$$
\psi(\mathbf{r}, \mathbf{R})=O_{n}(\mathbf{r}) P(\mathbf{r}-\mathbf{R}),
$$

where $P$ is the illumination function on the sample, $r$ is a vector in the plane perpendicular to the optical axis at the sample, $R$ is the relative shift between the sample and the illumination function, and $n$ is the iteration number. This is propagated to the Fraunhofer plane where, as is usual in iterative methods, the modulus is replaced by the recorded data and the phase is preserved. Upon backpropagation (center directed arrows in Fig. 2), the resulting exit wave differs from the initial exit wave estimate. The difference between these is used to make an update to the current estimate of the object function, weighted according to the modulus and phase of the illuminating function at each point over the object, such that

$$
O_{n+1}(\mathbf{r})=O_{n}(\mathbf{r})+U(\mathbf{r}-\mathbf{R})\left[\psi_{c, n}(\mathbf{r}, \mathbf{R})-\psi_{n}(\mathbf{r}, \mathbf{R})\right],
$$

where the subscript $\psi_{c}$ refers to the corrected exit wave after backpropagation and $U$ is the weight function of the update; for further details, please refer to Ref. [12]. As subsequent data from adjoining areas of the object are fed into the algorithm, a solution quickly appears: within only a few iterations. This is thousands of times faster than conventional iterative methods $[7,19]$. The speed of con- vergence, which can be measured using a least-squares estimate of the inconsistency between the calculated and experimental diffraction patterns [12], is derived from the increased quantity of data relative to unknowns and the fact that our knowledge of the aperture displacement implicitly yields ptychographical phases. We call this processing method a ptychographical iterative engine (PIE) [10]. The essential constraint that is being used is that the illumination function, whatever its modulus or phase structure, remains constant as it is scanned across the object. It

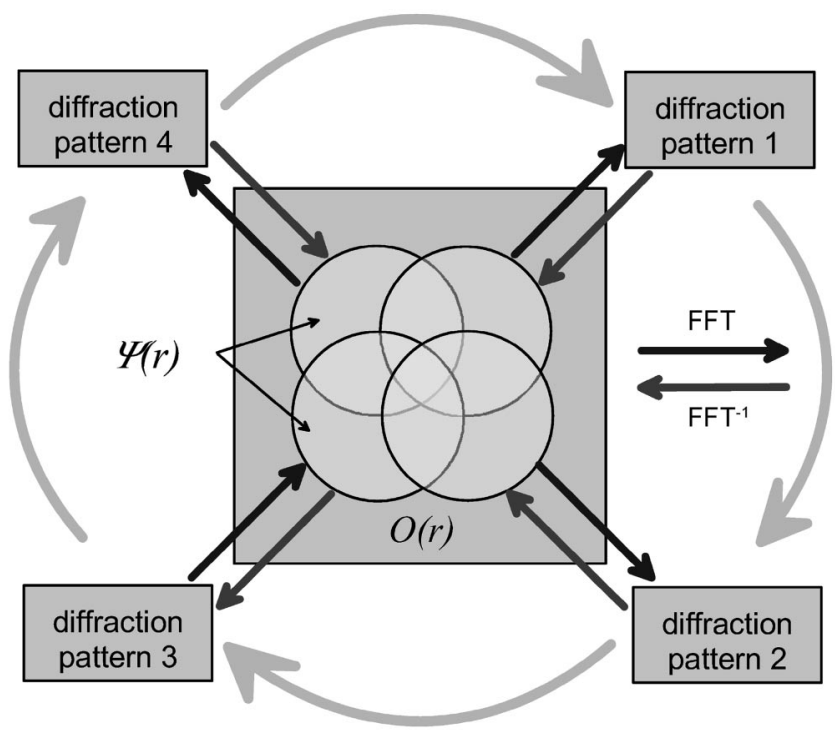

FIG. 2. Diagram of the phase-retrieval algorithm. The outer circular arrows indicate the position stepping within one iteration. The arrows within indicate (inverse) Fourier transforms and the desired input-output information. 
is well known that it is difficult for conventional phaseretrieval methods to settle on the position of the object within the depth of the illumination field [19]. In the present context, diffracted data from several illumination positions will lead only to a self-consistent solution at one well-defined value of defocus (i.e., the plane of the object), and hence this defocus ambiguity does not arise in the PIE method.

Experimental. -Modern third-generation synchrotron sources provide enough x-ray flux and coherence for the PIE algorithm. The experiment described in this Letter was carried out at the microXAS beam line of the Swiss Light Source (Paul Scherrer Institut, Switzerland), with an X-ray energy of $8.0 \mathrm{keV}(\lambda=0.155 \mathrm{~nm})$. A liquid nitrogen cooled Si (111) double reflection monochromator was used. The beam defining pinhole aperture $(5.0 \mu \mathrm{m}$ diameter, composed of Pt/Ir; see Fig. 1) was placed at a distance of $35 \mathrm{~m}$ from the undulator source. In view of earlier measurements with similar illumination optics [20], we infer that the radiation transmitted through the aperture was substantially, although not perfectly, spatially coherent. The sample was placed $1.1 \mathrm{~mm}$ downstream from the aperture so that the sample could be easily moved or changed. The transmission through the aperture and $1.1 \mathrm{~mm}$ of free space propagation defined the illumination function $(P)$. The entire data process has been assembled as a movie [21]. An estimate of the complex illumination function was calculated using Fresnel propagation between the aperture and the object. Unlike the Fienup hybridinput-output algorithm [2], the PIE algorithm can accommodate softly varying illumination fields which have both modulus and phase variations. The sample, ironically a gold Fresnel zone plate (of thickness $1 \mu \mathrm{m}$ ), was illuminated in $17 \times 17$ overlapping positions that were spaced by $2.5 \mu \mathrm{m}$, nine of which are shown in Fig. 3(b). Thus, the total field of view on the sample in this experiment covers a $45 \times 45 \mu \mathrm{m}^{2}$ area. The diffraction patterns (also shown in Fig. 3) were recorded with a fiber coupled CCD detector (Photonic Science Hystar, effective pixel size $4.5 \mu \mathrm{m}$ ) located $1.7 \mathrm{~m}$ downstream from the sample. Each pattern was recorded twice: a short $(8 \mathrm{~s})$ exposure to capture the intense central region near the unscattered beam and a longer ( $80 \mathrm{~s}$ ) exposure to record higher $q$ values. To begin the iterative reconstruction, a constant modulus and phase map was assumed for the initial estimate of the object. This object was then locally updated as the PIE algorithm swept through the matrix of diffraction patterns, following Eq. (2). We make no assumptions about the structure or scattering characteristics of the sample. The reconstructed modulus and phase of the object after 50 iterations is shown in Figs. 3(c) and 3(d), respectively. On the outskirts of the zone plate, the alternating attenuating and phase shifting concentric rings in the center of the image, is a random distribution of gold balls ranging from 250 to $1500 \mathrm{~nm}$ in diameter. Both the ring structure and the out-
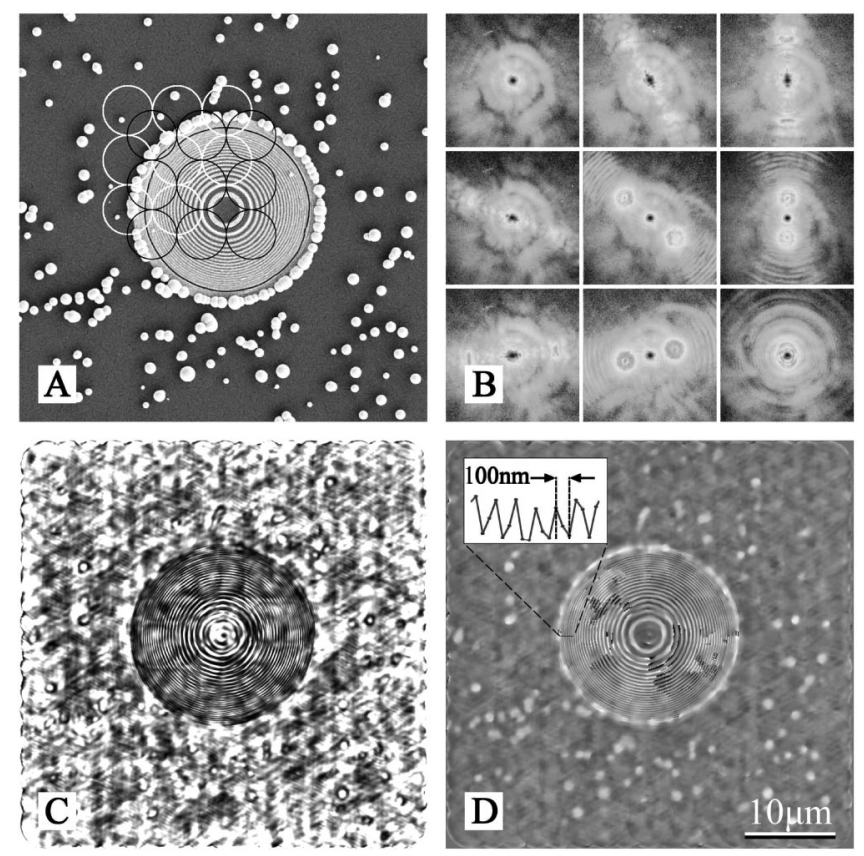

FIG. 3. Experimental results for a test sample with gold structures. (a) A scanning electron micrograph of the test sample with gold nanostructures. The circles indicate 18 of the 289 probe positions for which diffraction patterns were recorded. (b) Diffraction patterns for the positions indicated by white circles in (a). Subplots (c) and (d) are the reconstructed modulus and phase images displayed on a linear gray scale ranging from 0 to 1 and from $-\pi$ to $\pi$, respectively. The inset in (d) is a profile through the outermost rings of the zone plate which have a width of $100 \mathrm{~nm}$. The moire fringes visible in (d) are caused by the image compression necessary for reproduction but are not visible in the original full-size reconstruction.

lying gold balls are clearly visible in both the reconstructed modulus and the phase. The phase variation across the zone plate has a peak-to-peak amplitude close to the expected value of $0.62 \pi$ (calculated from the expected phase shift due to this thickness of gold), although it wraps from $\pi$ to $-\pi$ at some places. The amplitude variation displays some anomalies, especially the bright intensity which occurs at the center of some of the gold particles: This may arise from errors in the specimen actuators (which we know have absolute accuracy of almost $1 \mu \mathrm{m}$ ) or residual partial coherence in the beam. Resolution in these images is estimated to be about $50 \mathrm{~nm}$ and was obtained by examining the image along a line perpendicular to the rings of the zone plate, shown in the inset in Fig. 3(d). Furthermore, the reconstruction of the randomly distributed gold balls demonstrates that this method is not limited to periodic structures.

Conclusions. - We have presented a new x-ray microscopy technique that can be used to directly explore extended biological and material science samples in their natural environment. We believe that this method can be readily and routinely applied to life science microscopy, 
e.g., to image cells or cellular substructures at $50 \mathrm{~nm}$ resolution or better. The technique can, in principle, deliver wavelength-limited resolution without the use of any highprecision optical elements, although to achieve this ultimate goal will require a very high brightness source (so that appreciable intensity will be scattered to large angles), of the sort contemplated for "fourth-generation" light sources. Experimentally, it eases and speeds up the preparation of samples; the use of hard $\mathrm{x}$ rays removes the necessity to work in vacuum. It offers the ability to locate the region of interest, which is currently a unique feature of this method, since all other state-of-the-art lensless techniques require the knowledge of the region of interest, the isolation of the sample, constraints around the sample, or crystalline structures. It is also not hindered by a "defocus ambiguity" or limited to a restricting depth of focus. This "iterative but directive" approach is computationally thousands of times faster than other leading iterative techniques and opens a new era to diffractive on-line microscopy and 3D tomography.

We gratefully acknowledge the assistance of D.K. Satapathy, X. Donath, B. Meyer, and D. Grolimund during the experiments. A.C.H. is supported by an EPSRC doctoral training account. J. M. R. and A. G. C. were supported in part by the proof-of-principle fund of the University of Sheffield. This work was performed at the Swiss Light Source, Paul Scherrer Institut, Villigen, Switzerland.

*To whom all correspondence should be addressed. Electronic address: j.m.rodenburg@shef.ac.uk

[1] R. W. Gerchberg and W. O. Saxton, Optik (Jena) 35, 237 (1972).

[2] J. R. Fienup, Appl. Opt. 21, 2758 (1982).
[3] J. Miao et al., Nature (London) 400, 342 (1999).

[4] J. Miao et al., Proc. Natl. Acad. Sci. U.S.A. 100, 110 (2003).

[5] J. M. Zuo, I. Vartanyants, M. Gao, R. Zhang, and L. A. Nagahara, Science 300, 1419 (2003).

[6] K. A. Nugent, A. G. Peele, H.N. Chapman, and A.P. Mancuso, Phys. Rev. Lett. 91, 203902 (2003).

[7] H. N. Chapman et al., J. Opt. Soc. Am. A 23, 1179 (2006).

[8] D. Shapiro et al., Proc. Natl. Acad. Sci. U.S.A. 102, 15343 (2005).

[9] S. Eisebitt et al., Nature (London) 432, 885 (2004).

[10] H. M. L. Faulkner and J. M. Rodenburg, Phys. Rev. Lett. 93, 023903 (2004).

[11] J. M. Rodenburg and H. M. L. Faulkner, Appl. Phys. Lett. 85, 4795 (2004).

[12] H. M. L. Faulkner and J. M. Rodenburg, Ultramicroscopy 103, 153 (2005).

[13] J. M. Rodenburg and H. M. L. Faulkner, U.K. Patent application No. PCT/GB2005/001464, "High resolution imaging" (2004).

[14] J.M. Rodenburg, A.C. Hurst, and A.G. Cullis, Ultramicroscopy 107, 227 (2007).

[15] W. Hoppe, Acta Crystallogr. Sect. A 25, 508 (1969).

[16] J. M. Rodenburg, Ultramicroscopy 27, 413 (1989).

[17] R. Hegerl and W. Hoppe, Ber. Bunsen-Ges. Phys. Chem. 74, 1148 (1970).

[18] J. M. Rodenburg and R. H. T. Bates, Phil. Trans. R. Soc. A 339, 521 (1992).

[19] P. Thibault, V. Elser, C. Jacobsen, D. Shapiro, and D. Sayre, Acta Crystallogr. Sect. A 62, 248 (2006).

[20] F. Pfeiffer et al., Phys. Rev. Lett. 94, 164801 (2005).

[21] See EPAPS Document No. E-PRLTAO-98-058705 for one video showing the reconstruction process proceeding over the first ten iterations and one showing the diffraction data, as the illumination is scanned across the specimen. For more information on EPAPS, see http://www.aip.org/ pubservs/epaps.html. 